\title{
Bounce and wormholes
}

\author{
N Pinto-Neto, F P Poulis and J M Salim \\ Centro Brasileiro de Pesquisas Físicas, Rua Xavier Sigaud, 150, CEP 22290-180, Rio \\ de Janeiro, Brazil. \\ E-mail: nelson.pinto@pq.cnpq.br, fppoulis@cbpf.br, jsalim@cbpf.br
}

\begin{abstract}
We investigate if theories yielding bouncing cosmological models also generate wormhole solutions. We show that two of them present sensible traversable static wormhole solutions, while for the third possibility such solutions are absent.
\end{abstract}

PACS numbers: 04.20.-q, 04.20.Ex, 04.20.Gz, 04.20.Jb 


\section{Introduction}

With the observation of the present acceleration of the Universe [1, it seems that fluids that violate the strong energy condition (SEC) [2] must indeed exist in Nature, and antigravitate. It is also speculated that such acceleration might also be driven by yet more exotic fluids, called phantom fields, which violate the weak energy condition (WEC), and may cause a future Big-Rip singularity [3]. These fluids can also be effectively obtained through non-minimal couplings between gravity and other fields [4], quantum corrections [5], or non-standard interactions among ordinary fields satisfying all the energy conditions [6].

Such kind of fluids may also play a fundamental role in the early Universe, either yielding an inflationary period [7], and/or avoiding the initial singularity as in the bouncing models [8]. Regarding the later possibility, it is also known that the violation of SEC may not be sufficient to produce a bounce in the past: some period of WEC violation is also required if the spatial sections have not positive curvature [9].

Fluids violating WEC are also necessary to obtain wormhole solutions [10] hence, in view of all those scenarios in which exotic sources are seriously taken into account, we can investigate in which of them traversable wormhole solutions can be obtained.

In the present paper we investigate the existence of wormhole solutions in theoretical frameworks which produce bouncing cosmological models. The existence and properties of these cosmological bounces have been studied in many papers [11, 12, 13, 14], but it remains to be investigated if such theoretical frameworks can produce wormhole solutions, which is a natural expectation to have due to the enormous parallel between bounces and wormholes (which are nothing but bounces in space).

Our work will be based in three models of bouncing universes. The first one considers a non-minimal coupling between electromagnetic and gravitational fields caused by quantum electrodynamics corrections to general relativity [11, 15], which could be related to magnetic fields observed in galaxies and intergalactic medium [16, 17]. This coupling leads to non-linearities in the theory and, consequently, violations of the energy conditions that enabled not only a bounce [11] but also a very nice traversable wormhole solution.

The next one considers a model of universe in a Weyl Integrable Space-time (WIST) [12], yielding an effective negative energy scalar field, which evolves from a Minkowski configuration in the infinite past, goes through a bounce and finish in another asymptotic flat universe. This pure geometric model can also give rise to a perfectly reasonable traversable wormhole.

The last case treated involves non-linear corrections to the electromagnetic field. Such corrections are needed when very intense fields are considered, where the particle creation phenomenon occurs. These non-linearities leads to the violation of energy conditions and allow a bounce [14] but not a wormhole. We will show that whenever one imposes the boundary conditions that characterizes the wormhole throat, the asymptotic behavior of the spacetime solution becomes unacceptable. Therefore, we 
have an example of a theoretically acceptable source that violates WEC and do not lead to a wormhole, showing that this condition is necessary but not sufficient to obtain such type of geometries.

Finally, we conclude that, although we have explicitly shown examples of wormholes solutions obtained using sources already present in other frameworks, this is not enough to consider such type of configuration as real as long as they are static. This still depends on dynamical treatments, which are beyond the scope of this paper.

\section{Non-minimal coupling between electromagnetic and gravitational fields}

Non-minimal coupling between electromagnetic and gravitational fields appears when one takes into account the vacuum polarization influence on the propagation of photons in a gravitational background using the one-loop approximation [15. In the present section, we will consider a non-minimal coupling term added to Einstein-Maxwell action for gravity. It was used to obtain a non singular cosmological model [11, to study the production of primordial magnetic fields [16, 17] and to yield inflation [18]. The complete lagrangian reads

$$
L=\sqrt{-g}\left[\frac{1}{2 k}\left(1+\lambda A_{\mu} A^{\mu}\right) R-\frac{1}{4} F_{\mu \nu} F^{\mu \nu}\right],
$$

where $k=8 \pi G, G$ is the Newton's constant, $\lambda$ is the non-minimal coupling constant, and $F^{\mu \nu}$ is the usual Maxwell tensor constructed from $A^{\mu}$. The variation of this Lagrangian with respect to $g_{\mu \nu}$ and $A^{\mu}$ yields the following equations:

$$
\left(1+\lambda A^{2}\right) G_{\mu \nu}+\lambda \square A^{2} g_{\mu \nu}-\lambda \nabla_{\nu} \nabla_{\mu} A^{2}+\lambda R A_{\mu} A_{\nu}=k T_{\mu \nu},
$$

and

$$
F^{\mu \nu} \| \nu_{\nu}=-\frac{\lambda}{k} R A^{\mu}
$$

where $G_{\mu \nu}$ is the usual Einstein tensor, $A^{2} \equiv A^{\mu} A_{\mu}$, and $T_{\mu \nu}$ is the Maxwell stress-energy tensor,

$$
T_{\mu \nu}=F_{\mu \alpha} F_{\nu}^{\alpha}+\frac{1}{4} g_{\mu \nu} F_{\alpha \beta} F^{\alpha \beta}
$$

We will look for solutions with the same source used in the cosmological solution obtained in Ref. [11], which presents a bounce, where $A_{\mu}=w_{\mid \mu}$. With this choice for the vector potential, it follows from (3) that $R=0$, and the set of equations reduces to

$$
\begin{aligned}
& R_{\mu \nu}=\frac{\Upsilon_{|\mu| \mid \nu}}{\Upsilon}, \\
& g^{\mu \nu} \Upsilon_{|\mu| \mid \nu}=0,
\end{aligned}
$$

in which the last one comes from the trace of (2) and we have introduced the new variable $\Upsilon \equiv 1+\lambda A^{2}$. 
We are interested in a static spherical geometry, with metric given by

$$
d s^{2}=-e^{2 \phi(r)} d t^{2}+\frac{d r^{2}}{\left(1-\frac{b(r)}{r}\right)}+r^{2} d \Omega^{2} .
$$

The field equations (5) and (6), in this case, reduce to

$$
\begin{aligned}
& -\left(1-\frac{b}{r}\right) \frac{\phi^{\prime} \Upsilon^{\prime}}{\Upsilon}=\frac{b^{\prime}}{r} \\
& \frac{1}{\Upsilon} \sqrt{1-\frac{b}{r}}\left(\sqrt{1-\frac{b}{r}} \Upsilon^{\prime}\right)^{\prime}=-\frac{b}{r^{3}}+2\left(1-\frac{b}{r}\right) \frac{\phi^{\prime}}{r} \\
& \left(1-\frac{b}{r}\right) \frac{\Upsilon^{\prime}}{r \Upsilon}=\left(1-\frac{b}{r}\right)\left[\phi^{\prime \prime}+\phi^{\prime}\left(\phi^{\prime}+\frac{1}{r}\right)\right]-\frac{\left(b^{\prime} r-b\right)}{2 r^{2}}\left(\phi^{\prime}+\frac{1}{r}\right), \\
& \frac{\sqrt{1-\frac{b}{r}}}{r^{2} e^{\phi}}\left(r^{2} e^{\phi} \sqrt{1-\frac{b}{r}} \Upsilon^{\prime}\right)^{\prime}=0 .
\end{aligned}
$$

This last one implies that

$$
r^{2} e^{\phi} \sqrt{1-\frac{b}{r}} \Upsilon^{\prime}=D
$$

a constant, so that (9) can be rewritten as

$$
\frac{D}{\Upsilon} \sqrt{1-\frac{b}{r}}\left(\frac{1}{r^{2} e^{\phi}}\right)^{\prime}=-\frac{b}{r^{3}}+2\left(1-\frac{b}{r}\right) \frac{\phi^{\prime}}{r} .
$$

A simple exact solution to this non-linear system of differential equations reads

$$
\begin{aligned}
& b(r)=r_{0}, \\
& \phi(r)=\phi_{0}, \\
& \Upsilon(r)=\frac{2 D}{r_{0} e^{\phi_{0}}} \sqrt{1-\frac{r_{0}}{r}} .
\end{aligned}
$$

Let us now examine the properties and traversability of this wormhole, following the lines of [10]. The embedding function $z(r)$ reads

$$
z(r)=2 r_{0}\left(\frac{r}{r_{0}}-1\right)^{1 / 2}
$$

showing that it is a parabolic wormhole. The proper distance is given by

$$
l(r)=\sqrt{r} \sqrt{r-r_{0}}-\frac{r_{0}}{2} \ln \left(\frac{\sqrt{r}-\sqrt{r-r_{0}}}{\sqrt{r}+\sqrt{r-r_{0}}}\right),
$$

which tends towards $r$ when $r$ is big. 
As long as $\phi$ is constant, one can traverse the wormhole with constant speed, and the tidal radial acceleration is null. For the tidal lateral acceleration to be less than one Earth gravity, one has the condition on the speed $v$

$$
\frac{r_{0} \gamma^{2}}{2 r^{3}} \frac{v^{2}}{c^{2}} \geqslant \frac{1}{\left(10^{10} \mathrm{~cm}\right)^{2}},
$$

where $\gamma$ is the usual relativistic $\gamma$-factor, which we are assuming to be approximately equal to one, since we are considering non-relativistic velocities. Its maximum value is at the minimum of the throat at $r_{0}$, which implies that

$$
v \leqslant 42 \mathrm{~m} / \mathrm{s} \frac{r_{0}}{10 \mathrm{~m}}
$$

If the trip through the wormhole begins and ends in stations where the curvature of the wormhole is negligible, with deviations, say, of $0.01 \%$ from flatness, then they must be located at coordinate distances of order $r \approx 10^{4} r_{0}$, yielding a total proper distance of $\Delta l \approx 2 \times 10^{4} r_{0}$. This gives a total time travel of

$$
\Delta t=\frac{\Delta l}{v} \geqslant 1 \mathrm{~h} 23 \mathrm{~min} \text {. }
$$

For a one year travel one needs

$$
v \approx 6.25 r_{0} 10^{-4} / \mathrm{s} .
$$

Note that the electromagnetic energy required to construct this wormhole is null because $F_{\mu \nu}$, and hence $T_{\mu \nu}$, are null.

\section{Effective negative energy scalar field}

As mentioned in the Introduction, cosmological bounces can also be induced by negative energy fluids [19, 13, 20]. We will consider in this section a negative energy stiff matter fluid represented by a massless free scalar field, which can appear due to interactions among positive energy fluids [6], or through a pure geometrical model where spacetime is represented by a manifold with a geometric structure determined by the Weyl geometry [12]. In this later case, the model is empty, it starts as a flat Minkowski spacetime which, through instabilities, begin to collapse into a homogeneous and isotropic Weyl geometry.

The dynamics of the model is obtained from the action

$$
S=\int \sqrt{-g}\left(\hat{R}+\xi \hat{\nabla}_{\alpha} W^{\alpha}\right)
$$

where $\hat{R}$ is the Ricci tensor in the Weyl geometry, $W^{\alpha}$ is the Weyl vector satisfying $\hat{\nabla}_{\alpha} g_{\mu \nu}=W_{\alpha} g_{\mu \nu}$, and $\hat{\nabla}_{\alpha}$ is the covariant derivative with the Weyl affinity.

Taking a Weyl integrable space-time, where the Weyl vector is a gradient given by $W_{\lambda}=\varphi_{\mid \lambda}$, and performing the variation of the action $S$ with respect to the pair 
$\left(g_{\mu \nu}, \varphi\right)$ of independent variables yields the following equations of motion in terms of Riemannian geometrical quantities (see [12] for details):

$$
\begin{aligned}
G_{\mu \nu} & =-\lambda^{2}\left(\varphi_{\mid \mu} \varphi_{\mid \nu}-\frac{1}{2} \varphi_{\mid \alpha} \varphi^{\mid \alpha} g_{\mu \nu}\right) \equiv-\lambda^{2} T_{\mu \nu}^{\varphi}, \\
\square \varphi & =0,
\end{aligned}
$$

in which $\lambda^{2}=(4 \xi-3) / 2$. Note that if this constant is positive, one has an effective negative energy fluid, which gives rise to cosmological bounces [12, 13, 19, 20].

We are looking for the possibility of a static wormhole solution for the above set of equations. Using the spherical geometry given by the metric (7), the equations (24) and (25), after a simple manipulation, read

$$
\begin{aligned}
& b^{\prime}=-\frac{\lambda^{2}}{2}\left(1-\frac{b}{r}\right)\left(\varphi^{\prime}\right)^{2} r^{2}, \\
& \phi^{\prime}=\frac{b-\frac{r^{3} \lambda^{2}}{2}\left(1-\frac{b}{r}\right)\left(\varphi^{\prime}\right)^{2}}{2 r(r-b)}, \\
& {\left[\frac{1}{2}\left(1-\frac{b}{r}\right)\left(\varphi^{\prime}\right)^{2}\right]^{\prime}=-\left(1-\frac{b}{r}\right)\left(\varphi^{\prime}\right)^{2} \phi^{\prime}-\frac{2}{r}\left(1-\frac{b}{r}\right)\left(\varphi^{\prime}\right)^{2} .}
\end{aligned}
$$

This last one can be rewritten as

$$
\left[\left(r^{2} e^{\phi}\right)^{2}\left(1-\frac{b}{r}\right)\left(\varphi^{\prime}\right)^{2}\right]^{\prime}=0
$$

Now it is easy to integrate the resulting system of equations. The solution is:

$$
\begin{aligned}
& \varphi(r)=-\frac{\sqrt{2}}{\lambda} \arctan \left(\frac{r_{0}}{\sqrt{r^{2}-r_{0}^{2}}}\right)+\frac{\pi \sqrt{2}}{2 \lambda}+\varphi\left(r_{0}\right), \\
& b(r)=\frac{r_{0}^{2}}{r} \\
& \phi(r)=\phi_{0} .
\end{aligned}
$$

The space-time generated by this solution is represented by the geometry described by the following metric:

$$
d s^{2}=-e^{2 \phi_{0}} d t^{2}+\frac{d r^{2}}{\left(1-\frac{r_{0}^{2}}{r^{2}}\right)}+r^{2} d \Omega^{2} .
$$

As it was done in the previous section, let us examine the properties and traversability of this wormhole, again following the lines of [10]. The embedding function $z(r)$ now reads

$$
z(r)=r_{0} \operatorname{arccosh}\left(\frac{r}{r_{0}}\right)
$$


showing that it is a hyperbolic wormhole. The proper distance is given by

$$
l(r)=r \sqrt{1-\left(\frac{r_{0}}{r}\right)^{2}},
$$

which, again, tends towards $r$ when $r$ is big. Also, as long as $\phi$ is constant, one can traverse the wormhole with constant speed, and the tidal radial acceleration is null. For the tidal lateral acceleration to be less than one Earth gravity, one has the condition on the maximum speed $v$ at $r=r_{0}$ inside the throat,

$$
v \leqslant 30 \mathrm{~m} / \mathrm{s} \frac{r_{0}}{10 \mathrm{~m}} .
$$

For a $0.01 \%$ departure from flatness at the stations, they must be located at coordinate distances of order $r \approx 10^{2} r_{0}$, yielding a total proper distance of $\Delta l \approx$ $2 \times 10^{2} r_{0}$. This gives a total time travel of

$$
\Delta t=\frac{\Delta l}{v} \geqslant 67 \mathrm{~s}
$$

For a one year travel one needs

$$
v \approx 6.25 r_{0} 10^{-6} / \mathrm{s}
$$

In this case, some (negative) energy for the scalar field is required to construct this wormhole as long as its energy momentum tensor $T_{\mu \nu}^{\varphi}$ is not null. The energy density is $\rho=-r_{0}^{2} / r^{4}$, which in the throat at $r=r_{0}$ reaches its maximum value $\rho=-1 / r_{0}^{2}$, and the total (negative) mass of the scalar field reads $M=-2 \pi^{2} r_{0}$. Hence, smaller values of $r_{0}$ requires less mass but bigger densities inside the throat.

\section{Non-linear electrodynamics}

In [14] bouncing solutions where obtained considering a generalization of Maxwell electrodynamics, given by non-linear local covariant and gauge-invariant terms which depend on field invariants up to second order, as the source of classical Einstein's equations. This modification is expected to be relevant when the fields reach large

values, as occurs in the early universe. One expects that such modifications may also be important inside the hypothetical throat of a wormhole, generated by this source.

The generalization of Maxwell electrodynamics, up to second order, is determined by the following lagrangian [14]

$$
L=-\frac{1}{4} F+\alpha F^{2}+\beta G^{2}
$$

where

$$
F:=F_{\mu \nu} F^{\mu \nu}
$$

and

$$
G:=\frac{1}{2} \eta_{\alpha \beta \mu \nu} F^{\alpha \beta} F^{\mu \nu}
$$


where $\alpha$ and $\beta$ are arbitrary constants and $\eta_{\alpha \beta \mu \nu}$ is a totally anti-symmetric tensor. According to that same reference, in the early universe, the source should be identified with a hot primordial plasma. And, to keep isotropy, spatial average should be taken into account [21]. This can be done by associating the following mean values to the electric $(\boldsymbol{E})$ and magnetic $(\boldsymbol{H})$ fields.

$$
\begin{aligned}
& \overline{E_{i}}=0, \quad \overline{H_{i}}=0, \quad \overline{E_{i} H_{j}}=0, \\
& \overline{E_{i} E_{j}}=-\frac{1}{3} E^{2} g_{i j}, \\
& \overline{H_{i} H_{j}}=-\frac{1}{3} H^{2} g_{i j},
\end{aligned}
$$

according to the definition

$$
\bar{C} \equiv \lim _{V \rightarrow V_{0}} \frac{1}{V} \int C \sqrt{-g} d^{3} x^{i}
$$

to an arbitrary quantity $C$, in which $V=\int \sqrt{-g} d^{3} x^{i}$ and $V_{0}$ represents a sufficiently large time dependent three-volume.

The high temperature of the plasma led us to consider the situation where only the squared magnetic field $H^{2}$ survives and the electric field is put to $E^{2}=0$. The resulting averaged energy-momentum tensor is identified as a perfect fluid with modified expressions for the energy density $\rho$ and pressure $p$ given by

$$
\begin{aligned}
& \rho=\frac{H^{2}}{2}\left(1-8 \alpha H^{2}\right), \\
& p=\frac{H^{2}}{6}\left(1-40 \alpha H^{2}\right) .
\end{aligned}
$$

This source violates the energy condition $\rho+3 p \geqslant 0$, as in the models presented before. Nonetheless, it is worth to call attention that, in this case, the pressure is isotropic. We will look for a static solution in a spherical geometry given by the metric (7). The field equations become:

$$
\begin{aligned}
b^{\prime} & =\rho r^{2}, \\
\phi^{\prime} & =\frac{b+p r^{3}}{2 r^{2}\left(1-\frac{b}{r}\right)}, \\
p^{\prime} & =-(\rho+p) \phi^{\prime} .
\end{aligned}
$$

Eliminating $\phi^{\prime}$ from these equations we obtain

$$
\begin{aligned}
& b^{\prime}=\rho r^{2} \\
& p^{\prime}=-(\rho+p) \frac{\left(b+p r^{3}\right)}{2 r^{2}\left(1-\frac{b}{r}\right)} .
\end{aligned}
$$

The boundary conditions at the wormhole's throat, $r_{0}$, are 


$$
\begin{array}{ll}
b_{0} \equiv b\left(r_{0}\right)=r_{0}, & b_{0}^{\prime} \equiv b^{\prime}\left(r_{0}\right) \leqslant 1, \\
p_{0} \equiv p\left(r_{0}\right)=-\frac{1}{r_{0}^{2}}, & p_{0}^{\prime} \equiv p^{\prime}\left(r_{0}\right)=\frac{b_{0}^{\prime}-3}{r_{0}^{3}}<0,
\end{array}
$$

which imply for $X(r) \equiv H^{2}(r)$ :

$$
X_{0} \equiv X\left(r_{0}\right)=\frac{1+\sqrt{1+\gamma}}{80 \alpha} \geqslant \frac{1}{16 \alpha}, \quad X_{0}^{\prime} \equiv X^{\prime}\left(r_{0}\right)>0
$$

where $\gamma=960 \alpha / r_{0}^{2}$.

The system (49) becomes

$$
\begin{aligned}
& b^{\prime}=\frac{X}{2}(1-8 \alpha X) r^{2}, \\
& X^{\prime}=\frac{X(16 \alpha X-1)\left(40 \alpha X^{2} r-X r-\frac{6 b}{r^{2}}\right)}{\left(1-\frac{b}{r}\right)(80 \alpha X-1)},
\end{aligned}
$$

and any solution of it with the above boundary conditions has the property that the radial coordinate possesses a minimum value at $r_{0}$. However, for this case, these boundary conditions turn the solution completely unacceptable as we move away from the throat. We can see this by examining the behavior of $X(r)$. This function begins with a positive value and increases, so it must reach a maximum value, $X_{m} \equiv X\left(r_{m}\right)>$ $X_{0}$, at $r_{m}>r_{0}$ if we want the functions $p(r), \rho(r), b(r)$ and $\phi(r)$ to remain finite. Otherwise, the resulting space-time would not be acceptable. Imposing that $b(r) / r$ be finite (requirement of asymptotic flatness), we can see by (53) that $X(r)$ will only stop increasing if one of these equations holds true:

$$
\begin{aligned}
& X_{m}=0, \\
& 16 \alpha X_{m}-1=0, \\
& 40 \alpha X_{m}^{2} r_{m}-X_{m} r_{m}-6 \frac{b_{m}}{r_{m}^{2}}=0,
\end{aligned}
$$

with $b_{m} \equiv b\left(r_{m}\right)$.

It is clear that the first one cannot be true, for it would imply that $X_{m}<X_{0}$. This same argument discards the second one, which states that $X_{m}=1 / 16 \alpha \leqslant X_{0}$ and not greater. The last one gives

$$
X_{m}=\frac{1+\sqrt{1+960 \alpha \frac{b_{m}}{r_{m}^{3}}}}{80 \alpha}=\frac{1+\sqrt{1+\gamma \frac{r_{0}^{2} b_{m}}{r_{m}^{3}}}}{80 \alpha},
$$

which is also smaller than $X_{0}$, since it is given by (52), and $b_{m} \leqslant r_{m}$ and $r_{0}<r_{m}$ implies that $r_{0}^{2} b_{m} / r_{m}^{3}<1$.

Therefore, $X(r)$ diverge positively, leading the quantities $p(r), \rho(r), b(r)$ and $\phi(r)$ to diverge negatively, which would imply in a infinitely negative mass and a horizon for large values of $r$. The only case in which $X(r)$ (and the other functions) can stop increasing is if $b(r)$ increases in such a way that $b(r) / r$ do also diverges and makes 
$X^{\prime}(r)=0$, which is not compatible with the requirement of asymptotic flatness. Hence we can conclude that any attempt to impose a minimum value for the radial coordinate in this case result in a too much problematic space-time for it to be considered a satisfactory solution. This case does not allow a reasonable wormhole solution.

\section{Conclusion}

In this paper we have investigated the existence of wormhole solutions in theories which produce cosmological bounces. We have shown that in one of them, concerning non-linear electrodynamics, no wormhole solution is possible, showing that it is not mandatory that violation of WEC, or theories which produce cosmological bounces, can also result in wormholes.

In the other two theories we investigated, we were able to obtain simple, traversable (in the sense of [10]) wormhole solutions with very nice properties. Both depend on a a parameter $r_{0}$, which cannot be very big in order to not impose unattainable speeds or a very long time to traverse the wormhole (see equations (22) and (38)).

In the case of section 2, non-minimal coupling between gravity and electromagnetism, one arrives at the nice situation that no electromagnetic energy (in the usual sense) is required to produce the wormhole. The parameter $r_{0}$ appears only in the denominator of the amplitude of the vector potential (see (16) and the definition of $\Upsilon$ ). However, as there is another arbitrary constant present in (16), one can adjust it to satisfy physical requirements while preserving values of $r_{0}$ compatible with traversability with reasonable speeds.

In the case of section 3 , the parameter $r_{0}$ appears in the energy density of the field (in the denominator), and in its total mass (in the numerator). Hence, a small $r_{0}$ would imply a small total quantity of scalar field, which is good, but a high concentration of the field in the throat. However, if this field does not interact with matter and other fields, the traveller could pass through the throat without any harm.

Finally, we would like to point out that the solutions obtained here are all static, as if those wormholes always existed. In order to see if they can really arise, it is necessary to consider a dynamical geometry. The resulting equations and solutions should then show if some initial configuration could evolve into a wormhole, and if it will be stable. It should also be possible to investigate if during the bouncing period, where WEC is only preserved when spacial sections have positive curvature, wormholes would have time to arise or not.

We have shown that the existence of static wormholes is indeed a perfectly plausible theoretical possibility, in reasonable theoretical frameworks. Their production in the

history of the universe or even in the laboratory depend on dynamical developments of the present investigation, which will be the subject of our future investigations. 


\section{Acknowledgments}

We acknowledge CNPq and CAPES for financial support.

\section{References}

[1] Perlmutter S et al 1998 Nature (London) 39151

Riess A et al 1998 Astron. J. 1161009

[2] Barceló C and Visser M 2002 Int. J. of Mod. Phys. D 111553

[3] Caldwell R R, Kamionkowski M and Weinberg N N 2003 Phys. Rev. Lett. 91071301

[4] Fewster C J and Osterbrink L W 2006 Phys. Rev. D 74044021

Carvalho F C and Saa A 2004 Phys. Rev. D 70087302

[5] Nojiri S, Odintsov S D and Sami M 2006 Phys. Rev. D 74046004

Alam U and Sahni V 2006 Phys. Rev. D 73, 084024

Pinto-Neto N and Santini E S 2003 Phys. Lett. A 31536

[6] Pinto-Neto N and Fraga B M O Cosmic acceleration from interaction of ordinary fluids Preprint arXiv:0711.3602

[7] Starobinsky A A 1979 Pis'ma Zh. Eksp. Teor. Fiz. 30719 [1979 JETP Lett. 30 682]

Mukhanov V and Chibisov G 1981 JETP Lett. 33532

Guth A 1981 Phys. Rev. D 23347

Linde A 1982 Phys. Lett. B 108389

[8] Tolman R C 1931 Phys. Rev. 381758

Murphy G 1973 Phys. Rev. D 84231

Melnikov V and Orlov S 1979 Phys. Lett A 70263

Barros J A, Pinto-Neto N and Sagioro-Leal M A 1998 Phys. Lett. A 241229

Colistete Jr. C, Fabris J C and Pinto-Neto N 2000 Phys. Rev. D 62083507

[9] Peter P and Pinto-Neto N 2002 Phys. Rev. D 65023513

[10] Morris M S and Thorne K S 1988 Am. J. of Phys. 56395

[11] Novello M and Salim J M 1979 Phys. Rev. D 20377

[12] Novello M, Oliveira L A R, Salim J M and Albaz E 1993 Int. J. of Mod. Phys. D 1641

[13] Peter P and Pinto-Neto N 2002 Phys. Rev. D 66063509

[14] De Lorenci V A, Klippert R, Novello M and Salim J M 2002 Phys. Rev. D 65063501

[15] Drummond I T and Hathrell S J 1980 Phys. Rev. D 22343

[16] Prokopec T Cosmological magnetic fields from photon coupling to fermions and bosons in inflation Preprint arXiv:astro-ph/0106247 and references therein

Prokopec T and Puchwein E 2004 Phys. Rev. D 70043004 and references therein

[17] Turner M S and Widrow L M 1988 Phys. Rev. D 372743

[18] Golovnev A, Mukhanov V and Vanchurin V Vector Inflation Preprint arXiv:0802.2068

[19] Finelli F, Peter P and Pinto-Neto N Spectra of primordial fluctuations in two-perfect-fluid regular bounces Preprint arXiv:0709.3074

[20] Bozza V and Veneziano G 2005 Phys. Lett. B 625177

[21] Tolman R C and Ehrenfest P 1930 Phys. Rev. 361791 